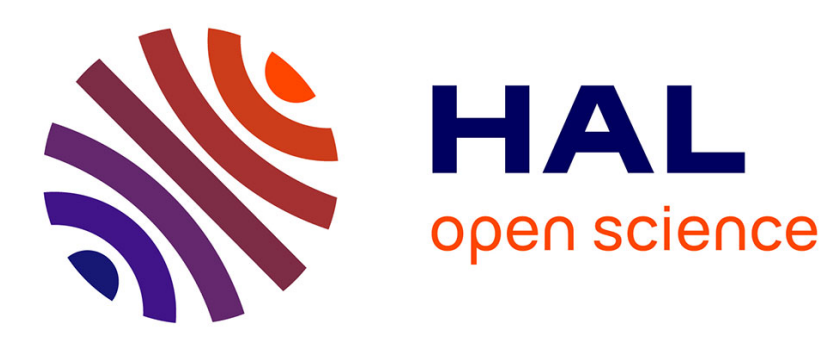

\title{
How cells sense their own shape - mechanisms to probe cell geometry and their implications in cellular organization and function
}

\author{
Armin Haupt, Nicolas Minc
}

\section{- To cite this version:}

Armin Haupt, Nicolas Minc. How cells sense their own shape - mechanisms to probe cell geometry and their implications in cellular organization and function. Journal of Cell Science, 2018, 131 (6), $10.1242 /$ jcs.214015 . hal-01864137

\section{HAL Id: hal-01864137 \\ https://hal.science/hal-01864137}

Submitted on 29 Aug 2018

HAL is a multi-disciplinary open access archive for the deposit and dissemination of scientific research documents, whether they are published or not. The documents may come from teaching and research institutions in France or abroad, or from public or private research centers.
L'archive ouverte pluridisciplinaire HAL, est destinée au dépôt et à la diffusion de documents scientifiques de niveau recherche, publiés ou non, émanant des établissements d'enseignement et de recherche français ou étrangers, des laboratoires publics ou privés. 
How Do Cells Sense their Own Shapes? : Mechanisms for Probing

Cell Geometry and their Implications in Cellular Organization and

\author{
Function
}

Armin Haupt and Nicolas Minc*

Institut Jacques Monod, CNRS UMR7592 and Université Paris Diderot, 15 rue Hélène Brion, 75205 Paris Cedex 13, France

*Correspondence to nicolas.minc@ijm.fr (N.M) 


\begin{abstract}
Cells come in a range of shapes that most often underlie their functions. Regulation of cell morphogenesis implicates that there are mechanisms for shape sensing which still remain poorly appreciated. Global and local cell geometrical features, such as aspect ratio, size or membrane curvature, may be probed by intracellular modules, such as the cytoskeleton, reaction-diffusion systems or molecular complexes. In multicellular tissues, cell shape emerges as an important means to transduce tissue-inherent chemical and mechanical cues into intracellular organization. One emergent paradigm is that cell shape sensing is most often based upon mechanisms of self-organization, rather than determinism. Here, we review relevant work that has elucidated some of the core principles of how cellular geometry may be conveyed into spatial information to guide processes, such as polarity, signaling, morphogenesis and division-plane positioning.
\end{abstract}




\section{INTRODUCTION}

Any biologist would acknowledge that cells are beautiful objects. The range of forms they span is not only aesthetic, but reveals the complexity and diversity of intracellular systems that serve to construct cell shape and, in most instances, reflects the function of the cell. Long polarized nerve cells make distant connections in brains and muscles; the small and flat shape of red blood cells may enhance flowing capacities needed to navigate in thin blood vessels; cuboidal epithelial cells serve as brick units for the walls of our organs; and cork-screw like bacteria can bore their way through the surface of epithelia. Besides the cell-type specific relevance of cell shape, an emergent concept is that both the regulation and functional use of cell shape, necessarily implicate geometry-sensing mechanisms. A cell that grows into a rodshape must come with designs that informs on the straightness of its long axis, or with signaling systems that detect any unwanted curvature. A symmetrically dividing cell needs to precisely locate its geometrical center and orient the division plane accounting for its global surface morphology.

According to their specific shapes, sizes and functions, cells may thus be equipped with internal systems to monitor global, local or dynamic geometrical features (Fig. 1). Those could confer robustness to cell shape, or act as means to amplify a small surface deformation, when shape changes are needed. Mechanisms that sense the straightness or curvature of the cell surface may be important to stabilize elongated cell shapes, or to detect local 3D membrane geometries, such as protrusions, or cilia (Cannon et al., 2017). Monitoring of the surface-to-volume ratio is thought to be important for timing cell division, or as means to locally titrate molecular reactions around cells (Harris and Theriot, 2016; Schmick and Bastiaens, 2014). Furthermore, the global cell aspect ratio has key relevance for the positioning and orientation of the division plane and in the definition of internal polarity axes (Minc et al., 2009; Minc et al., 2011) (Fig. 1).

Biologists have long recognized the relevance of feedback mechanisms that allow cells to probe their shapes and dimensions, as a basal property of all cells, even the roundest one (Gerhart, 1989; Hertwig, 1884a; Hertwig, 1884b; Moseley and Nurse, 2010; Wilson, 1925). Recent work has begun to decipher the generic designs and molecular mechanisms implicated. Dissecting specific mechanisms of geometry sensing in vivo may prove difficult, given the contributions from both chemical and mechanical cues, which also affect cell shapes in tissues. In vivo, for instance, processes such as polarity or division orientation have most often been linked to external signals, rather than as direct consequences of cell shape (Minc 
and Piel, 2012). However, it is becoming increasingly evident that cellular components such as the cytoskeleton and reaction-diffusion systems have self-organizing properties that can probe cellular boundary conditions (Karsenti, 2008). In addition, the advent of microfabrication methods to control and manipulate shapes independently of external cues in cells ranging from microbes to mammalian cells (Lautenschlager and Piel, 2013), has served as a driving force to unequivocally demonstrate that shape has a profound impact on the spatial organization and function of most cells.

We will here focus on key questions underlying the mechanisms of shape-sensing, and on the functional interplays between shape and internal organization: How do molecules and/or molecular assemblies probe cellular shapes? How do they use such information to control cell behavior and morphogenesis? Is cell-shape sensing a conserved trait in evolution needed for fitness? We will introduce the various geometrical parameters that may be sensed in different cells and discuss mechanisms, which link shape-sensing to the spatial control of processes, such as polarity, signaling, and division positioning. Although cell size is another important geometrical feature, we will not cover it here and refer the readers to recent reviews on this topic (Amodeo and Skotheim, 2016; Levy and Heald, 2015). 


\section{CELL SHAPE SENSING AND POLARITY}

\section{Microtubules as cell shape sensors for cell polarity}

Cell polarity underlies the ability of cells to define subcellular domains of activity at their surface. This allows cells to grow or migrate directionally, specify a front and a rear, or position internal organelles, such as the nucleus, the centrosome or the Golgi relative to each other (Etienne-Manneville, 2004). Polarization domains can spontaneously assemble as a result of positive feedbacks between interacting proteins and cytoskeleton elements that amplify local fluctuations into mature polar domains (Irazoqui et al., 2003; Wedlich-Soldner et al., 2003). Extrinsic or intrinsic chemical, mechanical or electrical cues, can then orient or stabilize those domains along specific directions (Casamayor and Snyder, 2002; EtienneManneville, 2004; Haupt et al., 2014; Ladoux et al., 2016).

As polarity ultimately directs processes such as cell growth or migration, it serves as a key input to define cell shapes over time. This has been extensively studied in the model fission yeast. These cells grow into a rod by targeting polarity and growth factors, such as the RhoGTPase Cdc42, actin and cell wall remodeling enzymes to cell tips (see (Chang and Martin, 2009) for a detailed review). Key to the tip-localization of polarity zones are dynamic microtubule (MT) bundles, which emanate from the nucleus and grow near parallel to the long cell axis to deposit MT-plus-end-associated landmark proteins at cell tips, which then recruit downstream elements. Mutants with defective MT organization have abnormal bent or branched shapes, and many mis-shaped mutants have defective MT organization, which has long suggested the existence of interplays between shape, MT organization and polarity (Verde et al., 1995).

Studies that manipulated fission yeast shape by physically bending cells in microfabricated devices have been instrumental in deciphering feedbacks between shape and polarity (Minc et al., 2009; Terenna et al., 2008). Subsequent to cell bending, MT organization dramatically changes, with most MTs not reaching the cell extremities anymore, but instead touching the cell sides, yielding the formation of ectopic polarization sites (Fig. 2A). In a reciprocal manner, round mutants with defective MT organization forced into a straight shape, recovered near wild-type MT orientation and polarization at cell tips (Terenna et al., 2008). Thus, MTs through their dynamic properties and straightness, serve as robust shape-sensors to promote polarization along a linear geometrical axis. This feedback between shape, MT orientation and polarity, may confer robustness to polarized growth and ensure the maintenance of rod- 
shape morphogenesis through generations of dividing cells (Drake and Vavylonis, 2013). Yet, polarizing spores or spheroplasts can readily elongate a first straight rod-shape even in the absence of MTs and landmarks; suggesting the existence of other systems promoting directed growth (Bonazzi et al., 2014; Kelly and Nurse, 2011).

Work on epithelial cells in fly embryonic tissues has suggested similar influence of cell shape on MT organization (Fig. 2B) (Gomez et al., 2016), with plausible implications on the definition of planar polarity axis (Butler and Wallingford, 2017). Alignment of MTs along the long axis has also been documented in adherent cells, and proposed to serve as a mechanism to limit the length of spreading cells (Levina et al., 2001; Picone et al., 2010). This effect may also contribute to guide the polarization of the nuclear-centrosome axis, to enhance persistent directional migration of cells which migrate along their long axis, although patterns of adhesion could serve as dominant spatial cues when cell geometry is mostly isotropic (Fig. 2B and C) (Jiang et al., 2005; McWhorter et al., 2013; Thery et al., 2006; Vignaud et al., 2012). Thus MT-based geometry-sensing may have broad relevance to polarity establishment and maintenance of eukaryotic cells.

\section{Subcellular micron-scale curvature sensing}

The rod-shape morphogenesis is also a common feature of many bacteria, including E. coli (Chang and Huang, 2014). In those cells, insertion of cell wall material occurs along the sides of the cylinder, not at cell poles, a process mediated by the actin homolog MreB (Furchtgott et al., 2011). A recent study found that MreB polymers tend to preferentially bind to regions of positive curvatures along the cylinder (Ursell et al., 2014). This observation supported a model, in which curvature detection and consequent local promotion of cell wall insertion and growth by MreB allowed cells to actively straighten their shape (Fig. 2D). However, a recent paper indicates that this feedback may not be sufficient to account for cell straightening upon larger deformation (Wong et al., 2017). Thus, bacterial cells, which lack long-range cytoskeletal systems, may ensure rod-shape maintenance through local curvature sensing, likely encoded in the structural properties of MreB polymers (Colavin et al., 2014).

Eukaryotic cells may also possess micron-scale curvature sensing systems. Septins are conserved oligomeric protein complexes that can assemble into non-polar filaments and bind to the cell cortex. They are important to many processes including cell shape, protein scaffolding and disease (Bridges and Gladfelter, 2015; Cannon et al., 2017). Yeast and 
mammalian septins have been recently demonstrated to have intrinsic affinities to bind surfaces with a defined positive curvature at the micron scale. Even though individual oligomers exhibit selective curvature binding, they require polymerisation to bind curved membranes within cells (Bridges et al., 2016). In cells, micron-scale positive curvature can be found on edges between spheres and protrusions (a topology often referred to as 'saddle', see Box 1), such as at the bases of dendritic spines, axons and cilia, in the yeast bud neck or branches of fungal hyphae, all structures where septins have been found to localize before (Fig. 2E) (Bridges and Gladfelter, 2015). The function of septins at some of these sites has been linked to a function as diffusion barriers or scaffolds (Hu et al., 2010; Merlini and Piatti, 2011). Thus, the inherent anisotropic curvature of saddle structures might promote the formation of specific higher-order septin organization, which is important for their biological functions.

This curvature-dependent septin recruitment might be used as a cellular readout. In budding yeast, the formation of a bud is monitored through the morphogenesis checkpoint that delays mitosis onset until bud emergence (Kang and Lew, 2017). The presumptive bud site is labelled by a septin ring, which might rearrange into higher-order structures that are aligned along the saddle that is formed upon bud emergence. This alignment could trigger the recruitment of Elm1, a kinase which influences mitotic entry (Fig. 2F) (Kang et al., 2016). Therefore, septin-mediated curvature sensing could also serve cells to monitor shape changes and time them with cell cycle progression.

Another prime example of local curvature sensing was recently proposed in the context of tip branching regulation during endothelial cell migration. Branching and general curvature formation in these cells was shown to be limited by acto-myosin cortex contraction (Elliott et al., 2015; Fischer et al., 2009). Myosin II was found to preferentially bind to flat membrane regions of low local curvature, with a binding efficiency that negatively correlates with absolute curvature. This generates a positive feedback loop, in which Myosin II may promote its own binding by actively reducing the curvature of bent membrane regions. In the context of branching morphogenesis, this feedback allows Myosin II to stabilize branches that form at small angles to pre-existing protrusions, while suppressing those that arise perpendicular to existing ones, thus polarizing branching along the migration direction (Fig. 2G). Curvaturesensing of Myosin was there again proposed to be encoded in the elongated morphology of filaments, which could favor their binding to actin on flat membranes (Elliott et al., 2015). Curvature dependence of acto-myosin forces has also been proposed to be important in the 
context of cytokinesis to promote faithful centripetal ring closure (Dorn et al., 2016; Zhou et al., 2015).

Evidence that cells may have developed ways to probe their local curvature on the micron scale through means other than direct polymer recognition of curved surfaces, has recently been obtained in fission yeast. In this system, the width of functional polarity domains scales with the local radius of curvature in rounded spores and rod-shaped cells (Bonazzi et al., 2015). This scaling was shown to rely on actin-dependent vesicle transport and fusion that may dilute and spread polar domains in a dose-dependent manner with the local curvature. According to this model, local geometry constrains could limit the volume that cortical actin cable networks can probe, yielding different cargo flux to dilute the cap (Fig. 2H). As polar domains built around conserved small GTPases such as Cdc42 usually scale to the size of cells ranging from small yeast to large eggs (Bonazzi et al., 2015; Jost and Weiner, 2015), it will be interesting to test if this curvature-sensing mechanism is based on similar actindependent transport processes in other cell types. Thus, the ability of actin or actinhomologues and their interactors to probe local micron-scale curvatures could serve as an important conserved shape-sensing design in morphogenesis.

\section{Local surface to volume ratio as a cue for polarization and signaling}

One interesting implication of the existence of flat, curved or narrow regions inside cells is that they may affect the local surface-to-volume ratio. The global surface-to-volume ratio, set by cell size, has been well documented to influence division timing or the scaling of internal organelles to cell size, but local effects are still poorly appreciated (Harris and Theriot, 2016; Wilbur and Heald, 2013). The impact of the local surface-to-volume ratio on local cell biochemistry was first explored theoretically using the example of a membrane bound kinase, which could phosphorylate a cytoplasmic substrate (Meyers et al., 2006). Competition between the kinase reaction rate and substrate diffusion results in a gradient of phosphorylation from the membrane to the cell center. A cell with a protruding flat front would in that context be able to set a different cytoplasmic biochemical activity, simply as a result of the increased local surface-to-volume ratio. This could in principle serve as a 'ruler' to measure cell shape and size (Fig. 2I) and could even recognize small membrane deformations. Yet, one important limitation of this model is that the lateral diffusion of the kinase in the membrane will rapidly disperse this gradient. As a consequence, this effect could 
only serve as a transient shape sensor, unless the kinase is continuously maintained in a polarized subcellular localization through reinforcements by cytoskeletal elements or other external cues (Schmick and Bastiaens, 2014). A recent study explored this mechanism in a systematic manner for reactions that occur within the membrane using an enzyme that diffuses in the cytoplasm or in the extracellular space, and proposed that a transient localized increase in reaction products could be achieved theoretically and experimentally, purely as a result of cell deformation, which even influenced signaling globally (Rangamani et al., 2013). Pioneering studies using micropatterned islands have long demonstrated that cell geometry could influence such important processes as differentiation, growth and death (Chen et al., 1997; Kilian et al., 2010; McWhorter et al., 2013). However, in this context, geometry was thought to indirectly affect the mechanical state of cells, which then influenced its fate (Chen et al., 1997). Thus, while the above-mentioned concept that cell shape could directly modulate signaling reactions await further generalization, it supports a provocative view that, at least in certain cell types, geometry per se could serve as a hub to mediate key cellular decision during tissue development and homeostasis (Rangamani et al., 2013; Ron et al., 2017; Schmick and Bastiaens, 2014).

\section{CELL SHAPE AND DIVISION POSITIONING}

\section{Cell shape and division in evolution}

Cell shape also has important influence on the positioning and orientation of the cell division plane, a process with profound implications for cell size control, stem cells and tissue morphogenesis (Minc and Piel, 2012). While some cells exhibit marked asymmetric divisions, most cells tend to divide in a symmetric manner by bisecting the mother cell into two halves of near exact same volume. Symmetric division is inherently linked to cell geometry, as here cells need to locate their geometrical center. In addition, apart from rare exceptions (Leisch et al., 2012), most elongated prokaryotic and eukaryotic cells tend to bisect their longest axis during symmetric division. This conserved behavior is fascinating in terms of evolution because mechanisms that position cytokinesis or septation vary largely between kingdoms (Oliferenko et al., 2009). In animal cells, cytokinesis is positioned from spatial cues that are provided by the mitotic spindle towards anaphase. In most fungi, cytokinesis and septum ingression are pre-specified from the position of the nucleus and the spindle usually aligns orthogonal to the septum. In plants, the preprophase band also forms around the nucleus and 
serve as a landmark for spindle orientation and cell plate growth. In bacteria and other prokaryotes, septum positional information arises from protein gradients in a manner mostly independent of DNA or any cytoskeletal structure (reviewed in Oliferenko et al., 2009).

These considerations raise the question of what kind of evolutionary pressure could have driven cells, which span a large range of size, shape, and environment, to divide along their long axis. One possibility is that this geometrical design could provide the largest cytoplasmic space for DNA segregation (Cadart et al., 2014; Lancaster et al., 2013). Another proposed idea is that this could promote the stability of ring position during ingression, at least in some cell types (Mishra et al., 2012). We also foresee that such a setting may minimize the possible error transfer between the detection of a geometrical center and the volume repartition between daughter cells. Finally, cell division along the long axis could just reflect inherent geometrical sensing properties of division-positioning machineries, which naturally tend to self-organize along the longest shape axis (Karsenti, 2008). Recent evo-devo studies suggest that variations in asymmetric division plane position are scaled with cell size, thereby ensuring a stable volume ratio between daughter cells (Farhadifar et al., 2015). Similar evodevo approaches assaying the variations in symmetric division position and orientation as a function of cell shape in closely related species should help address those important questions.

\section{Microtubule forces as geometrical rulers to target the cell center}

Historically, the relevance of cell shape for division positioning was first appreciated in large dividing animal embryos, leading to the formulations of early empirical "geometrical" rules for cell divisions (Hertwig, 1884a; Hertwig, 1893), see (Minc and Piel, 2012) for review. Indeed, most invertebrate and vertebrate zygotes cleave exactly in the cell center. This is challenging, because eggs are very large, up to a millimeter in amphibian species, and because fertilization, which brings centrosomes attached to the sperm pro-nucleus into the egg, is dramatically asymmetric. The questions thus arises of how can the nucleus and centrosome move to the exact geometric center of those large cells (Wuhr et al., 2009)? Apart from rare exceptions (see hereafter), nuclear centration in animal cells is mediated by MT-mediated forces, with actin being mostly dispensable. MTs nucleate around the centrosome and grow to form an aster, which then exerts forces to move the nucleus and centrosome to the cell center (Reinsch and Gonczy, 1998; Wuhr et al., 2009). 
While much debate is still ongoing on the exact nature and regulation of MT-mediated forces, one common well-accepted aspect of shape sensing and aster centration is that MT-exerted forces depend on average on the length of astral MTs. The combination of an aster-like geometry with the dynamic growth and shrinkage properties of MTs, and their lengthdependent forces provides a simple yet extremely robust design for the cell to be able to probe the cellular volume and center the centrosome (Fig. 3A) (Bornens, 2012; Holy et al., 1997; Kimura and Onami, 2005; Tanimoto et al., 2016; Wuhr et al., 2010). Length-scaling may arise from compressive pushing forces owing to MT-plus-end polymerization against the cell surface. Because of dynamic instabilities, more MTs will push on the centrosome as it is being brought closer to the cortex. Additionally, pushing may be limited by buckling in a length-dependent manner, so that longer MTs will buckle more than shorter ones, and these will thus exert comparatively more pushing forces on the centrosome (Fig. 3A) (Howard, 2006; Howard and Garzon-Coral, 2017). Indeed, such a mechanism has been demonstrated to promote nuclear centering in small yeast cells (Daga et al., 2006; Tolic-Norrelykke et al., 2005; Tran et al., 2001) and proposed to contribute to spindle centration in Caenorhabditis elegans (Garzon-Coral et al., 2016; Howard and Garzon-Coral, 2017). In vitro work and simulations suggest, however, that pushing could yield to unstable situations that tend to decenter asters if, for instance, MTs are allowed to pivot around centrosomes (Letort et al., 2016; Pinot et al., 2009). Whether those situations are relevant to cells remains to be tested.

In larger cells, such as eggs and early blastomeres of marine invertebrates, amphibians and fish, mounting evidence suggests that MT pushing is only poorly efficient in promoting aster centration (Hamaguchi and Hiramoto, 1986; Tanimoto et al., 2016; Wuhr et al., 2010). Length-scaling in these cells is thought to be driven from dynein motors that directly pull on MTs from sites in the cytoplasm (Barbosa et al., 2017; Hamaguchi et al., 1986; Kimura and Kimura, 2011; Longoria and Shubeita, 2013). The mechanical coupling of dyneins to the cytoplasm is thought to be mediated by the friction of cargos or endo-membranes that are moved by dyneins to the aster center. Accumulation of dynein-cargo complexes onto MTs in a length-dependent manner naturally results in MT-pulling forces that scale with the length of MTs; but even if those complexes are diluted in the aster, the pulling forces are still predicted to scale to the length of MTs (although in a non-linear manner) (Fig. 3A). It is still unclear though whether there are types of endo-membrane or cargo that are more suitable for MT forces in bulk cytoplasm, and how these cytoplasmic anchors may be recycled back to the surface to be able to continuously sustain this effect. 
MT-based centering systems have remarkable abilities in tracking the geometrical confines of a cell. For instance, sperm asters, which nucleate on the side of the egg at fertilization, can precisely target the center of large eggs in tens of minutes without any prior positional information. This is because they can continuously monitor the local cell geometry through MT growth, length-limitation by the cortex and length-dependent forces. This was clearly demonstrated by manipulations of egg shape and sites of sperm entry, which all led to astercentering paths with sharp turning points that reflected the local cell geometry explored by the aster during centration (Fig. 3B) (Tanimoto et al., 2016; Tanimoto and Minc, 2017). Another property of length-dependent MT forces, is that they act as elastic springs with respect to the geometry of the cell. In recent work, magnetic beads attached to centrosomes in C. elegans metaphase spindles allowed to apply external calibrated forces to asters (Garzon-Coral et al., 2016). Centrosomes moved away from the cell center to stop at a position that depended on the applied force and rapidly repositioned to the center after force cessation, much like a spring (Fig. 3C). The stiffness value of this spring is of key biological significance. A small stiffness would permit aster fluctuations, rotations or displacement owing to other cellular cues (e.g. an asymmetric cortical domain), whereas a high stiffness would tend to "freeze" the aster at the cell center (Howard and Garzon-Coral, 2017). How stiffness and overall mechanical properties of centering machineries vary with shape, size and among different cell types, and how they relate to aster dynamics and division phenotypes is an exciting future research direction.

Finally, it is worth noting that some cells may promote nuclear or centrosome centration relative to cell shape using systems that may not solely rely on MT forces. Actomyosin contraction might produce forces that could directly influence MT dynamics, aster motion and position (Burakov et al., 2003; Zhu et al., 2010). Remarkably, mouse oocytes, which lack centrosomes and asters, are capable of moving nuclei to the center of large eggs, albeit in a much slower manner than in embryos discussed above (Almonacid et al., 2015). This is thought to be mediated by a gradient of actin-driven cytoplasmic agitation, with more agitation close to the cell cortex. As a result of this gradient, a nucleus positioned off-center will experience more "kicks" on the cortex-facing side, which will move it to the center of the cell. Thus in some cases, actin may contribute to geometry sensing to move large structures and organelles to the center of cells. 


\section{Orienting division with cell shape}

After the nucleus has reached the center, the centrosome duplicates and defines an orientation axis for the mitotic spindles. As observed almost 150 years ago, this axis most often correlates with the long axis of the cell, but how cells probe their geometry to orient division accordingly remained mysterious. Recent studies of the cleavage patterns of early embryos suggested that length-dependent MT forces could robustly function in orientating the division axis with respect to cell shape anisotropies (Minc et al., 2011; Wuhr et al., 2010). In essence, the distribution of MT length around two sister asters generate torques that rotate the centrosome pair, with a favored configuration of minimal energy that corresponds to the long axis of the cell (Fig. 3D). This model was validated in multiple studies, including by manipulating the shape of embryos, or by exploiting the natural changes in 3D blastomere cell shapes that occur in various cleaving embryos (Bjerknes, 1986; Minc et al., 2011; Mitchison et al., 2012; Pierre et al., 2016; Wuhr et al., 2010). One prime example is the cleavage pattern of fish embryos, which entails a choreography of successive cell divisions with precise 3D angular settings (Fig. 3E). The one-cell fish egg consists of a flat blastodisc located on a large yolk layer. Because the yolk blocks aster growth, the aster adopts a flat 3D shape, and as a consequence the first division axis lies parallel to the yolk interface. At telophase, asters and centrosomes are duplicated and elongate along the long sister aster shapes, perpendicular to the first division axis, giving rise to a division orthogonal to the previous plane (Wuhr et al., 2010). This alternate orthogonal sequence of division axis and cell/aster shapes continues on until the 16-cell stage, at which point the four most-centered blastomeres, which are squeezed by their neighbors, elongate their shape perpendicular to the yolk and orient their division along this shape axis, thereby ensuring tissue layering in the $3^{\text {rd }}$ dimension (Olivier et al., 2010) (Fig. 3E). Strikingly, 3D models that are solely based on shape-sensing from lengthdependent MT forces were able to account for this cleavage pattern in fish and other species (Pierre et al., 2016). Thus, the interplay between shapes and division can serve as an iterative design to produce complex patterns of oriented divisions that are instrumental to the morphogenetic development of embryos and tissues (Pierre et al., 2016; Xiong et al., 2014).

In light of these models, the factors that contribute to shape cells are thus ultimately key to understand division positioning in multicellular tissues. Cortical tension and cell-cell adhesion are prime cell-shape regulators in tissues, but tissue tension or compression forces from neighboring cells could contribute as well (Fig. 3E and F). In return, the orientation of cell division along the cell shape axis also has functional implications in tissues. It may serve to 
relax stresses in epithelial monolayers (Campinho et al., 2013; Wyatt et al., 2015), or as the basis of an homeostatic mechanism regulating the topology of cell contacts (Gibson et al., 2011). One important current debate is to discern whether division orientation in tissues under tension or compression may be caused by the direct effect of external forces on the division machinery (Fink et al., 2011), or is the sole result of deformed cell shapes that orient division (Minc et al., 2011; Pierre et al., 2016).

Cell shape can also change during mitosis. Rounding is prominent in adherent vertebrate cells and in epithelia, and appears as one important mechanism to temporarily erase the contribution of geometry to division axis determination (Thery and Bornens, 2008). Yet, in many instances, the mitotic spindle in the rounded cell still aligns with the preceding interphase long axis. Seminal work using cells seeded on micro-patterns showed that this memory is provided by actin fibers, which read the geometry of the adhesive pattern and influence polarity cues inside the round mitotic cell to orient the spindle (Thery and Bornens, 2006; Thery et al., 2007; Thery et al., 2005). Studies of the protein Mud in fly epithelia (Numa in vertebrates) have recently suggested that similar shape-memory mechanisms exist in tissues (Bosveld et al., 2016). An interesting recent study also revealed that when rounding is inhibited in the fly embryo epithelium, cell shape will largely override cortical polarity cues, yielding division orientations that are oftentimes orthogonal to the epithelial layer (Chanet et al., 2017). Thus, a tentative speculation is that any significant anisotropy in cell shape could dominate over other signals in symmetrically dividing cells.

Cells lacking centrosomes such as the early blastomeres of many rodent embryos (Courtois et al., 2012) can still orient their division to cell shape by mechanisms that thus far remain mostly mysterious (Gray et al., 2004). Actin may be involved at some level, but the geometrical features that allow the actin cytoskeleton to read cell shape in this context are not known (Chaigne et al., 2016). Cortical and/or cytoplasmic protein gradients may also contribute to division positioning relative to cell shape. For instance, in adherent cells, gradients of Ran-GTP that emanate from chromosomes and diffusible signals from polo-like kinase that is located at spindle poles could serve as rulers to monitor and control the position and orientation of the spindle (Kiyomitsu and Cheeseman, 2012). In bacterial cells, dynamic gradients of the Min proteins serve as geometry sensors to promote medial bacterial fission (see (Kretschmer and Schwille, 2016) for a recent review). Bacterial systems have selforganized membrane-associated patterns that are built around a core set of three proteins, Min 
C, -D and -E, which exhibit pole-to-pole oscillations as a result of specific rules of their biochemical interactions and diffusion properties. Through time-averaging, this system defines a narrow mid-cell zone where the septum machinery is allowed to assemble to divide the cell. Remarkably, the Min proteins can self-assemble into dynamic patterns in vitro and probe the geometry to define a long axis even in triangular or squared shapes (Schweizer et al., 2012; Wu et al., 2015; Zieske and Schwille, 2014). Thus a system based on reactiondiffusion can generate specific positional protein patterns with respect to the geometry of a cell and may represent an additional important feature to connect cell shape with division position (Xiao et al., 2017).

\section{CONCLUSION AND FUTURE DIRECTIONS}

The "morphobiological" mechanisms that allow cells to probe their geometry are still at their premise stage. However, by summarizing recent efforts in the literature, we identified several generic design principles, which appear to have been repeatedly used in evolution. The first one is based on MT networks, which can convert cellular geometries into defined modes of polarization or oriented division. MTs serve as prominent rulers, primarily owing to the longrange and dynamic nature of MT polymers and the forces they exert on the cortex, the cytoplasm and on nuclei and spindles. A second more local design feature is based on the differential curvature recognition that is encoded in the structural properties of molecular assemblies, such as septins, MreB filaments, and Myosin filaments. The final feature is based on reaction-diffusion and its ability to form self-assembled patterns relative to cell geometry. An intriguing realization is that the above-mentioned systems exist in most cells, raising the question of how they may be tuned down to allow a more deterministic cue, such as for instance a chemical gradient, to override them.

Up to date, however, many of the relevant studies have only been performed at the single-cell level, oftentimes in model cell types, such as yeast, Hela cells, or invertebrate zygotes, with still only few studies having recognized the contribution of cell shape sensing in multicellular tissues. This is because tissues come with a plethora of cues that are frequently orienting cell behavior along the same direction as cell shape, making it difficult to disentangle them. In addition, cell shapes in tissues have complex 3D features, which are challenging to analyze. The standardization of novel microscopy methodologies, such as selective plane illumination microscopy (SPIM), or two-photon microscopy, could circumvent this limitation by allowing the analysis of cell shapes in 3 dimensions deep inside of developing tissues and organs. 
Finally, one area that is lagging behind in this field has been the systematic identification of the gene products specifically affected by cell shape, or required for shape-sensing. Given the advent of microfabrication methods, recent studies are now beginning to describe methods to systematically screen genes required for shape sensing, for instance during spindle orientation (Burri et al., 2017). Another way to approach this question is by exploiting cell shape variations, which are widespread in diseases such as cancer. To this end, the recent large-scale profiling of gene expression and image analysis pipelines has begun to establish dosedependent correlations between geometry and the activation of specific pathways in some cancers (Sailem and Bakal, 2017; Sero et al., 2015). Therefore, further investigating morphobiological mechanisms may also have relevance in understanding the contribution of cell geometry to the pathophysiological behavior of cells in disease conditions.

\section{ACKNOWLEDGMENT}

The authors acknowledge S. Dmitrieff for careful reading of the manuscript. Research in our laboratory is supported by the CNRS and grants from the "Mairie de Paris emergence" program, the FRM “amorçage" grant AJE20130426890, the ANR “CellSize” grant and the European Research Council (CoG Forcaster N 647073). 


\section{Text Box 1: Geometrical terms.}

Curvature: Defines how much a surface deviates from being flat. Throughout this review, we define curvature from the perspective of the cell interior. For a spherical cell this means that its inner surface has a negative curvature. A tube-shaped protrusion of this sphere also has a negative curvature along its transverse section, but furthermore generates a region of positive curvature at its base in the direction pointing from the sphere into the protrusion. The topology at the base is called a 'saddle', where the curvature along two orthogonal axes is opposite.

Aspect ratio: Defines the proportional relation of width and height of a two-dimensional object. It can be applied to cells if depth is similar to width or width has an approximately rotational symmetry along the height axis.

Surface-to-volume ratio: Defines the amount of surface area per unit volume of an object.

For an object with a given geometry, as it gets bigger, volume increases faster than the surface area. A spherical cell has the lowest surface-to-volume ratio, compared to e.g. flat or tube-like shapes.

\section{BIBLIOGRAPHY}

Almonacid, M., Ahmed, W. W., Bussonnier, M., Mailly, P., Betz, T., Voituriez, R., Gov, N. S. and Verlhac, M. H. (2015). Active diffusion positions the nucleus in mouse oocytes. Nat Cell Biol 17, 470-9.

Amodeo, A. A. and Skotheim, J. M. (2016). Cell-Size Control. Cold Spring Harb Perspect Biol 8, a019083.

Barbosa, D. J., Duro, J., Prevo, B., Cheerambathur, D. K., Carvalho, A. X. and Gassmann, R. (2017). Dynactin binding to tyrosinated microtubules promotes centrosome centration in C. elegans by enhancing dynein-mediated organelle transport. PLoS Genet 13, e1006941.

Bjerknes, M. (1986). Physical theory of the orientation of astral mitotic spindles. Science 234, 1413-6.

Bonazzi, D., Haupt, A., Tanimoto, H., Delacour, D., Salort, D. and Minc, N. (2015). ActinBased Transport Adapts Polarity Domain Size to Local Cellular Curvature. Curr Biol 25, 2677-83.

Bonazzi, D., Julien, J. D., Romao, M., Seddiki, R., Piel, M., Boudaoud, A. and Minc, N. (2014). Symmetry Breaking in Spore Germination Relies on an Interplay between Polar Cap Stability and Spore Wall Mechanics. Dev Cell 28, 534-46.

Bornens, M. (2012). The centrosome in cells and organisms. Science 335, 422-6.

Bosveld, F., Markova, O., Guirao, B., Martin, C., Wang, Z., Pierre, A., Balakireva, M., Gaugue, I., Ainslie, A., Christophorou, N. et al. (2016). Epithelial tricellular junctions act as interphase cell shape sensors to orient mitosis. Nature 530, 495-8.

Bridges, A. A. and Gladfelter, A. S. (2015). Septin Form and Function at the Cell Cortex. J Biol Chem 290, 17173-80. 
Bridges, A. A., Jentzsch, M. S., Oakes, P. W., Occhipinti, P. and Gladfelter, A. S. (2016). Micron-scale plasma membrane curvature is recognized by the septin cytoskeleton. J Cell Biol 213, 23-32.

Burakov, A., Nadezhdina, E., Slepchenko, B. and Rodionov, V. (2003). Centrosome positioning in interphase cells. J Cell Biol 162, 963-9.

Burri, O., Wolf, B., Seitz, A. and Gonczy, P. (2017). TRACMIT: An effective pipeline for tracking and analyzing cells on micropatterns through mitosis. PLOS ONE 12, e0179752.

Butler, M. T. and Wallingford, J. B. (2017). Planar cell polarity in development and disease. Nat Rev Mol Cell Biol 18, 375-388.

Cadart, C., Zlotek-Zlotkiewicz, E., Le Berre, M., Piel, M. and Matthews, H. K. (2014). Exploring the function of cell shape and size during mitosis. Dev Cell 29, 159-69.

Campinho, P., Behrndt, M., Ranft, J., Risler, T., Minc, N. and Heisenberg, C. P. (2013). Tension-oriented cell divisions limit anisotropic tissue tension in epithelial spreading during zebrafish epiboly. Nat Cell Biol 15, 1405-14.

Cannon, K. S., Woods, B. L. and Gladfelter, A. S. (2017). The Unsolved Problem of How Cells Sense Micron-Scale Curvature. Trends Biochem Sci 42, 961-976.

Casamayor, A. and Snyder, M. (2002). Bud-site selection and cell polarity in budding yeast. Curr Opin Microbiol 5, 179-86.

Chaigne, A., Campillo, C., Voituriez, R., Gov, N. S., Sykes, C., Verlhac, M. H. and Terret, M. E. (2016). F-actin mechanics control spindle centring in the mouse zygote. Nat Commun 7, 10253.

Chanet, S., Sharan, R., Khan, Z. and Martin, A. C. (2017). Myosin 2-Induced Mitotic Rounding Enables Columnar Epithelial Cells to Interpret Cortical Spindle Positioning Cues. Curr Biol 27, 33503358 e3.

Chang, F. and Huang, K. C. (2014). How and why cells grow as rods. BMC Biol 12, 54.

Chang, F. and Martin, S. G. (2009). Shaping fission yeast with microtubules. Cold Spring Harb Perspect Biol 1, a001347.

Chen, C. S., Mrksich, M., Huang, S., Whitesides, G. M. and Ingber, D. E. (1997). Geometric control of cell life and death. Science 276, 1425-8.

Colavin, A., Hsin, J. and Huang, K. C. (2014). Effects of polymerization and nucleotide identity on the conformational dynamics of the bacterial actin homolog MreB. Proc Natl Acad Sci U S A 111, 3585-90.

Courtois, A., Schuh, M., Ellenberg, J. and Hiiragi, T. (2012). The transition from meiotic to mitotic spindle assembly is gradual during early mammalian development. J Cell Biol 198, 357-70.

Daga, R. R., Yonetani, A. and Chang, F. (2006). Asymmetric microtubule pushing forces in nuclear centering. Curr Biol 16, 1544-50.

Dorn, J. F., Zhang, L., Phi, T. T., Lacroix, B., Maddox, P. S., Liu, J. and Maddox, A. S. (2016). A theoretical model of cytokinesis implicates feedback between membrane curvature and cytoskeletal organization in asymmetric cytokinetic furrowing. Mol Biol Cell 27, 1286-99.

Drake, T. and Vavylonis, D. (2013). Model of fission yeast cell shape driven by membranebound growth factors and the cytoskeleton. PLoS Comput Biol 9, e1003287.

Elliott, H., Fischer, R. S., Myers, K. A., Desai, R. A., Gao, L., Chen, C. S., Adelstein, R. S., Waterman, C. M. and Danuser, G. (2015). Myosin II controls cellular branching morphogenesis and migration in three dimensions by minimizing cell-surface curvature. Nat Cell Biol 17, 137-47.

Etienne-Manneville, S. (2004). Cdc42--the centre of polarity. J Cell Sci 117, 1291-300.

Farhadifar, R., Baer, C. F., Valfort, A. C., Andersen, E. C., Muller-Reichert, T., Delattre, M. and Needleman, D. J. (2015). Scaling, selection, and evolutionary dynamics of the mitotic spindle. Curr Biol 25, 732-40.

Fink, J., Carpi, N., Betz, T., Betard, A., Chebah, M., Azioune, A., Bornens, M., Sykes, C., Fetler, L., Cuvelier, D. et al. (2011). External forces control mitotic spindle positioning. Nat Cell Biol 13, 771-8.

Fischer, R. S., Gardel, M., Ma, X., Adelstein, R. S. and Waterman, C. M. (2009). Local cortical tension by myosin II guides 3D endothelial cell branching. Curr Biol 19, 260-5. 
Furchtgott, L., Wingreen, N. S. and Huang, K. C. (2011). Mechanisms for maintaining cell shape in rod-shaped Gram-negative bacteria. Mol Microbiol 81, 340-53.

Garzon-Coral, C., Fantana, H. A. and Howard, J. (2016). A force-generating machinery maintains the spindle at the cell center during mitosis. Science 352, 1124-7.

Gerhart, J. (1989). Topobiology. An Introduction to Molecular Embryology. Gerald M. Edelman. Basic Books, New York, 1988. xvi, 240 pp., illus. \$21.95. Science 243, 1373-4.

Gibson, W. T., Veldhuis, J. H., Rubinstein, B., Cartwright, H. N., Perrimon, N., Brodland, G. W., Nagpal, R. and Gibson, M. C. (2011). Control of the mitotic cleavage plane by local epithelial topology. Cell 144, 427-38.

Gomez, J. M., Chumakova, L., Bulgakova, N. A. and Brown, N. H. (2016). Microtubule organization is determined by the shape of epithelial cells. Nat Commun 7, 13172.

Gray, D., Plusa, B., Piotrowska, K., Na, J., Tom, B., Glover, D. M. and Zernicka-Goetz, M. (2004). First cleavage of the mouse embryo responds to change in egg shape at fertilization. Curr Biol 14, 397-405.

Hamaguchi, M. S., Hamaguchi, Y. and Hiramoto, Y. (1986). Microinjected polystyrene beads move along astral rays in sand dollar eggs. Development, growth \& differentiation 28, 461-470.

Hamaguchi, M. S. and Hiramoto, Y. (1986). Analysis of the Role of Astral Rays in Pronuclear Migration in Sand Dollar Eggs by the Colcemid-UV Method. Development, growth \& differentiation 28, 143-156.

Harris, L. K. and Theriot, J. A. (2016). Relative Rates of Surface and Volume Synthesis Set Bacterial Cell Size. Cell 165, 1479-1492.

Haupt, A., Campetelli, A., Bonazzi, D., Piel, M., Chang, F. and Minc, N. (2014).

Electrochemical regulation of budding yeast polarity. PLoS Biol 12, e1002029.

Hertwig, O. (1884a). Das Problem der Befruchtung und der Isotropie des Eies, eine Theory der Vererbung. Jenaische Zeitschrist.

Hertwig, O. (1884b). Das Problem der Befruchtung une der Isotropie des Eies, eine Theory der Vererbung. Jenaische Zeitschrist.

Hertwig, O. (1893). Ueber den Werth der ersten Furchungszellen fuer die Organbildung des Embryo. Experimentelle Studien am Frosch- und Tritonei. Arch. mikr. Anat. xlii 662-807.

Holy, T. E., Dogterom, M., Yurke, B. and Leibler, S. (1997). Assembly and positioning of microtubule asters in microfabricated chambers. Proc Natl Acad Sci U S A 94, 6228-31.

Howard, J. (2006). Elastic and damping forces generated by confined arrays of dynamic microtubules. Physical Biology 3, 54.

Howard, J. and Garzon-Coral, C. (2017). Physical Limits on the Precision of Mitotic Spindle Positioning by Microtubule Pushing forces: Mechanics of mitotic spindle positioning. Bioessays 39.

Hu, Q., Milenkovic, L., Jin, H., Scott, M. P., Nachury, M. V., Spiliotis, E. T. and Nelson, W. J. (2010). A septin diffusion barrier at the base of the primary cilium maintains ciliary membrane protein distribution. Science 329, 436-9.

Irazoqui, J. E., Gladfelter, A. S. and Lew, D. J. (2003). Scaffold-mediated symmetry breaking by Cdc42p. Nat Cell Biol 5, 1062-70.

Jiang, X., Bruzewicz, D. A., Wong, A. P., Piel, M. and Whitesides, G. M. (2005). Directing cell migration with asymmetric micropatterns. Proc Natl Acad Sci U S A 102, 975-8.

Jost, A. P. and Weiner, O. D. (2015). Probing Yeast Polarity with Acute, Reversible, Optogenetic Inhibition of Protein Function. ACS Synth Biol 4, 1077-85.

Kang, H. and Lew, D. J. (2017). How do cells know what shape they are? Curr Genet 63, 75

77.

Kang, H., Tsygankov, D. and Lew, D. J. (2016). Sensing a bud in the yeast morphogenesis checkpoint: a role for Elm1. Mol Biol Cell 27, 1764-75.

Karsenti, E. (2008). Self-organization in cell biology: a brief history. Nat Rev Mol Cell Biol 9, $255-62$.

Kelly, F. D. and Nurse, P. (2011). De novo growth zone formation from fission yeast spheroplasts. PLoS One 6, e27977. 
Kilian, K. A., Bugarija, B., Lahn, B. T. and Mrksich, M. (2010). Geometric cues for directing the differentiation of mesenchymal stem cells. Proc Natl Acad Sci U S A 107, 4872-7.

Kimura, A. and Onami, S. (2005). Computer simulations and image processing reveal lengthdependent pulling force as the primary mechanism for $\mathrm{C}$. elegans male pronuclear migration. Dev Cell 8, 765-75.

Kimura, K. and Kimura, A. (2011). Intracellular organelles mediate cytoplasmic pulling force for centrosome centration in the Caenorhabditis elegans early embryo. Proc Natl Acad Sci U S A 108, $137-42$.

Kiyomitsu, T. and Cheeseman, I. M. (2012). Chromosome- and spindle-pole-derived signals generate an intrinsic code for spindle position and orientation. Nat Cell Biol 14, 311-7.

Kretschmer, S. and Schwille, P. (2016). Pattern formation on membranes and its role in bacterial cell division. Curr Opin Cell Biol 38, 52-9.

Ladoux, B., Mege, R. M. and Trepat, X. (2016). Front-Rear Polarization by Mechanical Cues: From Single Cells to Tissues. Trends Cell Biol 26, 420-433.

Lancaster, O. M., Le Berre, M., Dimitracopoulos, A., Bonazzi, D., Zlotek-Zlotkiewicz, E., Picone, R., Duke, T., Piel, M. and Baum, B. (2013). Mitotic rounding alters cell geometry to ensure efficient bipolar spindle formation. Dev Cell 25, 270-83.

Lautenschlager, F. and Piel, M. (2013). Microfabricated devices for cell biology: all for one and one for all. Curr Opin Cell Biol 25, 116-24.

Leisch, N., Verheul, J., Heindl, N. R., Gruber-Vodicka, H. R., Pende, N., den Blaauwen, T. and Bulgheresi, S. (2012). Growth in width and FtsZ ring longitudinal positioning in a gammaproteobacterial symbiont. Curr Biol 22, R831-2.

Letort, G., Nedelec, F., Blanchoin, L. and Thery, M. (2016). Centrosome centering and decentering by microtubule network rearrangement. Mol Biol Cell 27, 2833-43.

Levina, E. M., Kharitonova, M. A., Rovensky, Y. A. and Vasiliev, J. M. (2001). Cytoskeletal control of fibroblast length: experiments with linear strips of substrate. J Cell Sci 114, 4335-41.

Levy, D. L. and Heald, R. (2015). Biological Scaling Problems and Solutions in Amphibians. Cold Spring Harb Perspect Biol 8, a019166.

Longoria, R. A. and Shubeita, G. T. (2013). Cargo transport by cytoplasmic dynein can center embryonic centrosomes. PLOS ONE 8, e67710.

McWhorter, F. Y., Wang, T., Nguyen, P., Chung, T. and Liu, W. F. (2013). Modulation of macrophage phenotype by cell shape. Proc Natl Acad Sci U S A 110, 17253-8.

Merlini, L. and Piatti, S. (2011). The mother-bud neck as a signaling platform for the coordination between spindle position and cytokinesis in budding yeast. Biol Chem 392, 805-12.

Meyers, J., Craig, J. and Odde, D. J. (2006). Potential for control of signaling pathways via cell size and shape. Curr Biol 16, 1685-93.

Minc, N., Bratman, S. V., Basu, R. and Chang, F. (2009). Establishing new sites of polarization by microtubules. Curr Biol 19, 83-94.

Minc, N., Burgess, D. and Chang, F. (2011). Influence of cell geometry on division-plane positioning. Cell 144, 414-26.

Minc, N. and Piel, M. (2012). Predicting division plane position and orientation. Trends Cell Biol 22, 193-200.

Mishra, M., Huang, Y., Srivastava, P., Srinivasan, R., Sevugan, M., Shlomovitz, R., Gov, N., Rao, M. and Balasubramanian, M. (2012). Cylindrical cellular geometry ensures fidelity of division site placement in fission yeast. J Cell Sci 125, 3850-7.

Mitchison, T., Wuhr, M., Nguyen, P., Ishihara, K., Groen, A. and Field, C. M. (2012). Growth, interaction, and positioning of microtubule asters in extremely large vertebrate embryo cells. Cytoskeleton (Hoboken) 69, 738-50.

Moseley, J. B. and Nurse, P. (2010). Cell division intersects with cell geometry. Cell 142, 184-

8.

Oliferenko, S., Chew, T. G. and Balasubramanian, M. K. (2009). Positioning cytokinesis. Genes Dev 23, 660-74. 
Olivier, N., Luengo-Oroz, M. A., Duloquin, L., Faure, E., Savy, T., Veilleux, I., Solinas, X., Debarre, D., Bourgine, P., Santos, A. et al. (2010). Cell lineage reconstruction of early zebrafish embryos using label-free nonlinear microscopy. Science 329, 967-71.

Picone, R., Ren, X., Ivanovitch, K. D., Clarke, J. D., McKendry, R. A. and Baum, B. (2010). A polarised population of dynamic microtubules mediates homeostatic length control in animal cells. PLOS Biol 8, e1000542.

Pierre, A., Salle, J., Wuhr, M. and Minc, N. (2016). Generic Theoretical Models to Predict Division Patterns of Cleaving Embryos. Dev Cell 39, 667-682.

Pinot, M., Chesnel, F., Kubiak, J. Z., Arnal, I., Nedelec, F. J. and Gueroui, Z. (2009). Effects of confinement on the self-organization of microtubules and motors. Curr Biol 19, 954-60.

Rangamani, P., Lipshtat, A., Azeloglu, E. U., Calizo, R. C., Hu, M., Ghassemi, S., Hone, J., Scarlata, S., Neves, S. R. and lyengar, R. (2013). Decoding information in cell shape. Cell 154, 135669.

Reinsch, S. and Gonczy, P. (1998). Mechanisms of nuclear positioning. J Cell Sci 111 ( Pt 16), 2283-95.

Ron, A., Azeloglu, E. U., Calizo, R. C., Hu, M., Bhattacharya, S., Chen, Y., Jayaraman, G., Lee, S., Neves-Zaph, S. R., Li, H. et al. (2017). Cell shape information is transduced through tensionindependent mechanisms. Nat Commun 8, 2145.

Sailem, H. Z. and Bakal, C. (2017). Identification of clinically predictive metagenes that encode components of a network coupling cell shape to transcription by image-omics. Genome Res 27, 196-207.

Schmick, M. and Bastiaens, P. I. H. (2014). The interdependence of membrane shape and cellular signal processing. Cell 156, 1132-1138.

Schweizer, J., Loose, M., Bonny, M., Kruse, K., Monch, I. and Schwille, P. (2012). Geometry sensing by self-organized protein patterns. Proc Natl Acad Sci U S A 109, 15283-8.

Sero, J. E., Sailem, H. Z., Ardy, R. C., Almuttaqi, H., Zhang, T. and Bakal, C. (2015). Cell shape and the microenvironment regulate nuclear translocation of NF-kappaB in breast epithelial and tumor cells. Mol Syst Biol 11, 790.

Tanimoto, H., Kimura, A. and Minc, N. (2016). Shape-motion relationships of centering microtubule asters. J Cell Biol 212, 777-87.

Tanimoto, H. and Minc, N. (2017). Quantitative approaches for the study of microtubule aster motion in large eggs. Methods Cell Biol 139, 69-80.

Terenna, C. R., Makushok, T., Velve-Casquillas, G., Baigl, D., Chen, Y., Bornens, M., Paoletti, A., Piel, M. and Tran, P. T. (2008). Physical mechanisms redirecting cell polarity and cell shape in fission yeast. Curr Biol 18, 1748-53.

Thery, M. and Bornens, M. (2006). Cell shape and cell division. Curr Opin Cell Biol 18, 648-57.

Thery, M. and Bornens, M. (2008). Get round and stiff for mitosis. HFSP J 2, 65-71.

Thery, M., Jimenez-Dalmaroni, A., Racine, V., Bornens, M. and Julicher, F. (2007). Experimental and theoretical study of mitotic spindle orientation. Nature 447, 493-6.

Thery, M., Racine, V., Pepin, A., Piel, M., Chen, Y., Sibarita, J. B. and Bornens, M. (2005). The extracellular matrix guides the orientation of the cell division axis. Nat Cell Biol 7, 947-53.

Thery, M., Racine, V., Piel, M., Pepin, A., Dimitrov, A., Chen, Y., Sibarita, J. B. and Bornens, M. (2006). Anisotropy of cell adhesive microenvironment governs cell internal organization and orientation of polarity. Proc Natl Acad Sci U S A 103, 19771-6.

Tolic-Norrelykke, I. M., Sacconi, L., Stringari, C., Raabe, I. and Pavone, F. S. (2005). Nuclear and division-plane positioning revealed by optical micromanipulation. Curr Biol 15, 1212-6.

Tran, P. T., Marsh, L., Doye, V., Inoue, S. and Chang, F. (2001). A mechanism for nuclear positioning in fission yeast based on microtubule pushing. J Cell Biol 153, 397-411.

Ursell, T. S., Nguyen, J., Monds, R. D., Colavin, A., Billings, G., Ouzounov, N., Gitai, Z., Shaevitz, J. W. and Huang, K. C. (2014). Rod-like bacterial shape is maintained by feedback between cell curvature and cytoskeletal localization. Proc Natl Acad Sci U S A 111, E1025-34. 
Verde, F., Mata, J. and Nurse, P. (1995). Fission yeast cell morphogenesis: identification of new genes and analysis of their role during the cell cycle. J Cell Biol 131, 1529-38.

Vignaud, T., Galland, R., Tseng, Q., Blanchoin, L., Colombelli, J. and Thery, M. (2012). Reprogramming cell shape with laser nano-patterning. J Cell Sci 125, 2134-40.

Wedlich-Soldner, R., Altschuler, S., Wu, L. and Li, R. (2003). Spontaneous cell polarization through actomyosin-based delivery of the Cdc42 GTPase. Science 299, 1231-5.

Wilbur, J. D. and Heald, R. (2013). Mitotic spindle scaling during Xenopus development by kif2a and importin alpha. Elife 2, e00290.

Wilson, E. B. (1925). The cell in development and heredity. . New-York: Macmillan.

Wong, F., Renner, L. D., Ozbaykal, G., Paulose, J., Weibel, D. B., van Teeffelen, S. and Amir, A. (2017). Mechanical strain sensing implicated in cell shape recovery in Escherichia coli. Nat Microbio/ 2, 17115.

Wu, F., van Schie, B. G., Keymer, J. E. and Dekker, C. (2015). Symmetry and scale orient Min protein patterns in shaped bacterial sculptures. Nat Nanotechnol 10, 719-26.

Wuhr, M., Dumont, S., Groen, A. C., Needleman, D. J. and Mitchison, T. J. (2009). How does a millimeter-sized cell find its center? Cell Cycle 8, 1115-21.

Wuhr, M., Tan, E. S., Parker, S. K., Detrich, H. W., 3rd and Mitchison, T. J. (2010). A model for cleavage plane determination in early amphibian and fish embryos. Curr Biol 20, 2040-5.

Wyatt, T. P., Harris, A. R., Lam, M., Cheng, Q., Bellis, J., Dimitracopoulos, A., Kabla, A. J., Charras, G. T. and Baum, B. (2015). Emergence of homeostatic epithelial packing and stress dissipation through divisions oriented along the long cell axis. Proc Natl Acad Sci U S A 112, 5726-31.

Xiao, S., Tong, C., Yang, Y. and Wu, M. (2017). Mitotic Cortical Waves Predict Future Division Sites by Encoding Positional and Size Information. Dev Cell 43, 493-506 e3.

Xiong, F., Ma, W., Hiscock, T. W., Mosaliganti, K. R., Tentner, A. R., Brakke, K. A., Rannou, N., Gelas, A., Souhait, L., Swinburne, I. A. et al. (2014). Interplay of cell shape and division orientation promotes robust morphogenesis of developing epithelia. Cell 159, 415-27.

Zhou, Z., Munteanu, E. L., He, J., Ursell, T., Bathe, M., Huang, K. C. and Chang, F. (2015). The contractile ring coordinates curvature-dependent septum assembly during fission yeast cytokinesis. Mol Biol Cell 26, 78-90.

Zhu, J., Burakov, A., Rodionov, V. and Mogilner, A. (2010). Finding the cell center by a balance of dynein and myosin pulling and microtubule pushing: a computational study. Mol Biol Cell 21, 4418-27.

Zieske, K. and Schwille, P. (2014). Reconstitution of self-organizing protein gradients as spatial cues in cell-free systems. Elife 3. 
FIGURES AND FIGURE LEGENDS

Geometrical feature

A

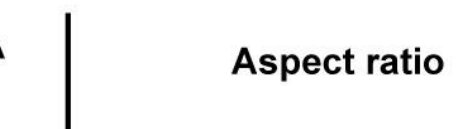

B

B

C

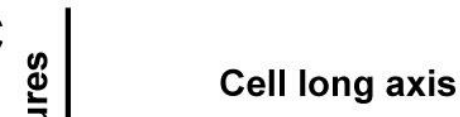

Curvature

Cell long axis

Surface/Volume ratio

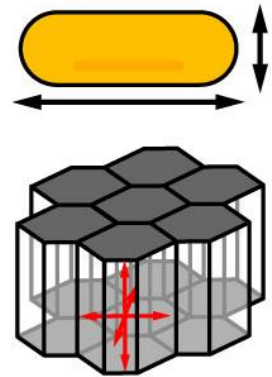

Example

Fission yeast

Epithelial cells

Vibrio cholerae

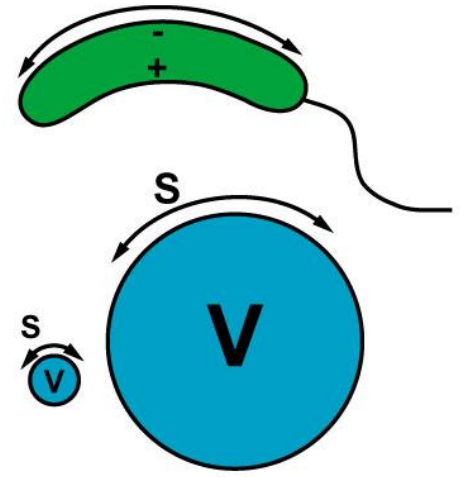

Bacterial

cocci

$\downarrow$ Oocyte

Migrating cell
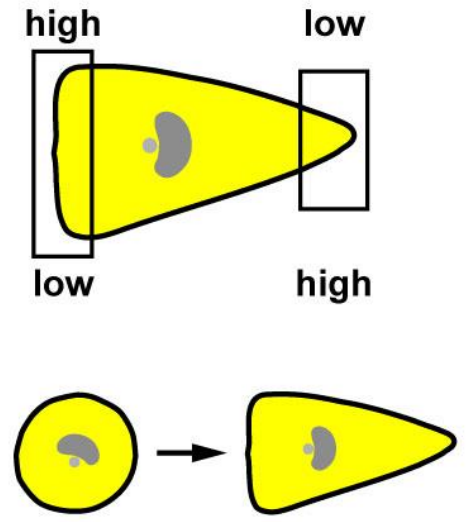

Migrating cell polarization

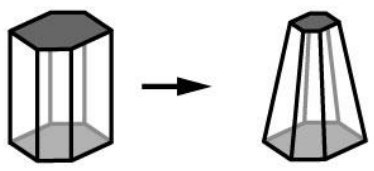

Fig. 1: Examples of cell shape features potentially used by cells to control behavior and organization. A) Global, cell-level features, such as aspect ratio, long axis, curvature and surface-to-volume ratio, might be monitored by a cell to ensure proper polarization, signaling 
or timing and positioning of cell division. Examples include microorganisms, epithelial cells and oocytes. B) Differences in local, subcellular geometrical features, such as curvature or surface-to-volume ratio, could be important to sustain cellular states, for instance during cell migration. C) Geometric features can dynamically change over time. During polarized cell migration, an initially isotropic cell has to develop a long axis. Another example is in the course of epithelial cell contraction, where the apical surface-to-volume ratio of a cell changes. 
Microtubule and polarity alignment with cell long axis

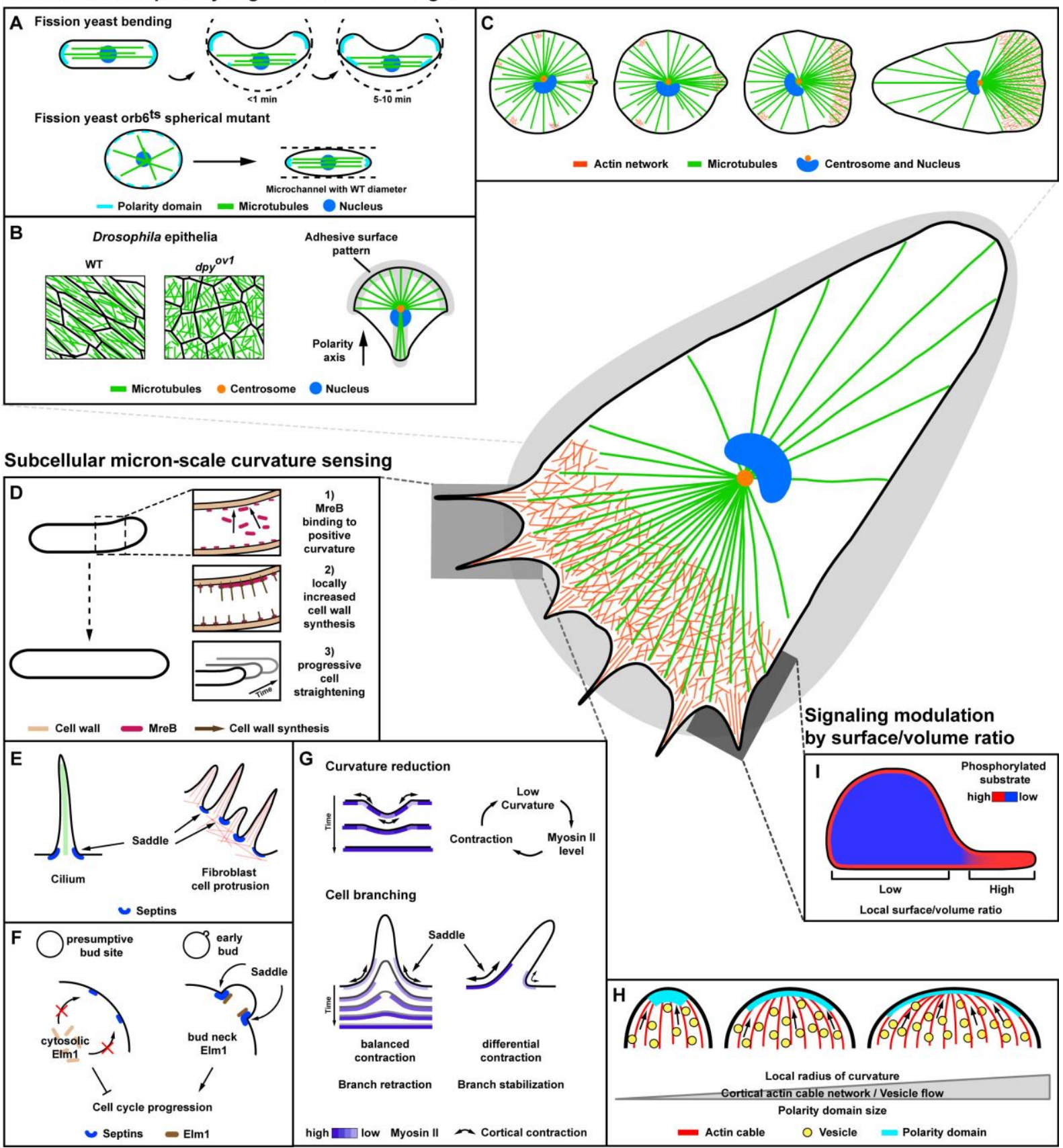

Fig. 2: Mechanisms of shape sensing with relevance for cell polarity, migration and

signaling. A) Fission yeast shape-sensing by MTs directly inputs on the positioning of polarity domains. B) Cell shape, MT alignment and polarization in animal cells. Left: Subapical MT alignment with cell shape in Drosophila epithelia. Right: In adherent cells, MTs organize along the long cell shape axis, defining the major polarity axis. Right panel is reproduced with modifications from (Thery et al., 2006), Copyright 2006 National Academy of Sciences. C) MTs dynamically align along the establishing long axis during symmetry breaking in cell migration. D) Bacterial shape-sensing and maintenance. E. coli cells detect shape defects through the preferential binding of MreB to positively curved membranes and 
straighten them through subsequent localized cell growth. E) Curvature sensing by septins through preferential binding to "saddle"-like membrane geometries, as found at the base of cilia or cell protrusions. F) Geometry-dependent septin organization, through curvature sensing, may allow yeast cells to monitor bud emergence. G) The binding of Myosin II to curved regions in endothelial cells is stabilized in a positive feedback loop, resulting in an overall curvature reduction and selective branch stabilization. H) In fission yeast spores, the size of actin networks and concomitant vesicle flow may scale with the local curvature radius, thereby ensuring that polarity domains adapt their size to local curvature. Reproduced with modifications from (Bonazzi et al., 2015), with permission from Elsevier. I) Local variations in surface-to-volume ratio could influence the kinetics of reactions between membrane-bound and cytoplasmic factors, thereby creating local biochemically distinct states, e.g. with regard to the phosphorylation status of a factor, around cells. 
Targeting the geometrical cell center

A
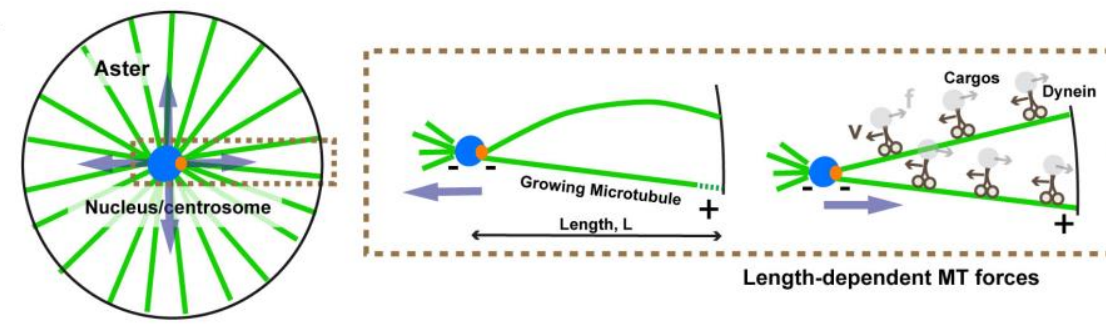
$\longleftarrow$ Length, $\mathrm{L}$ Length-dependent MT forces

B

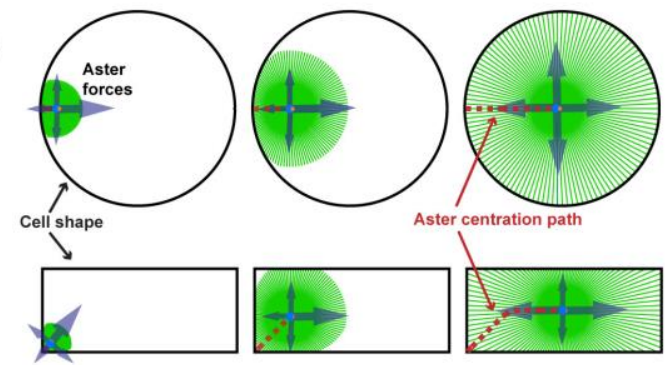

C

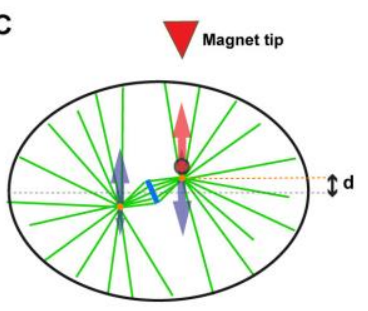

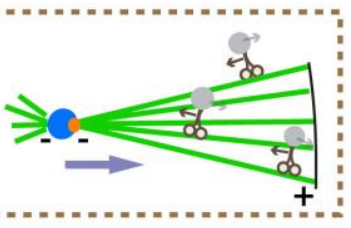
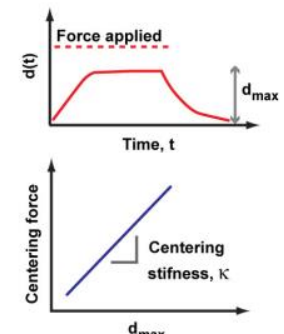

Dividing along the long axis

D
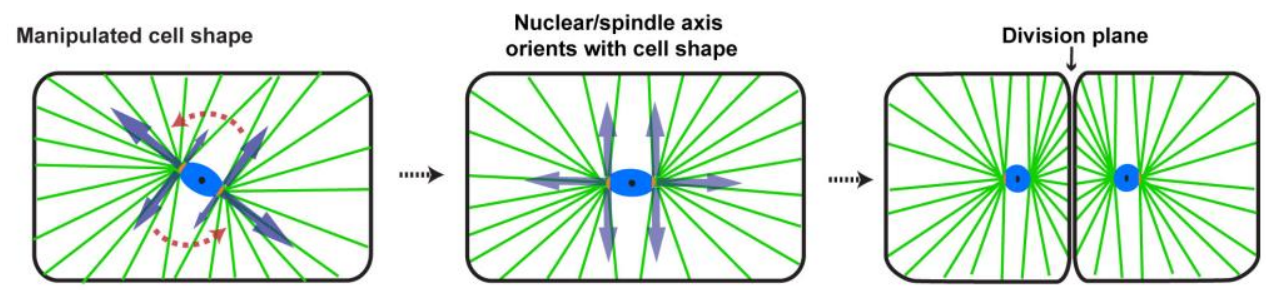

E

Natural shapes in tissue
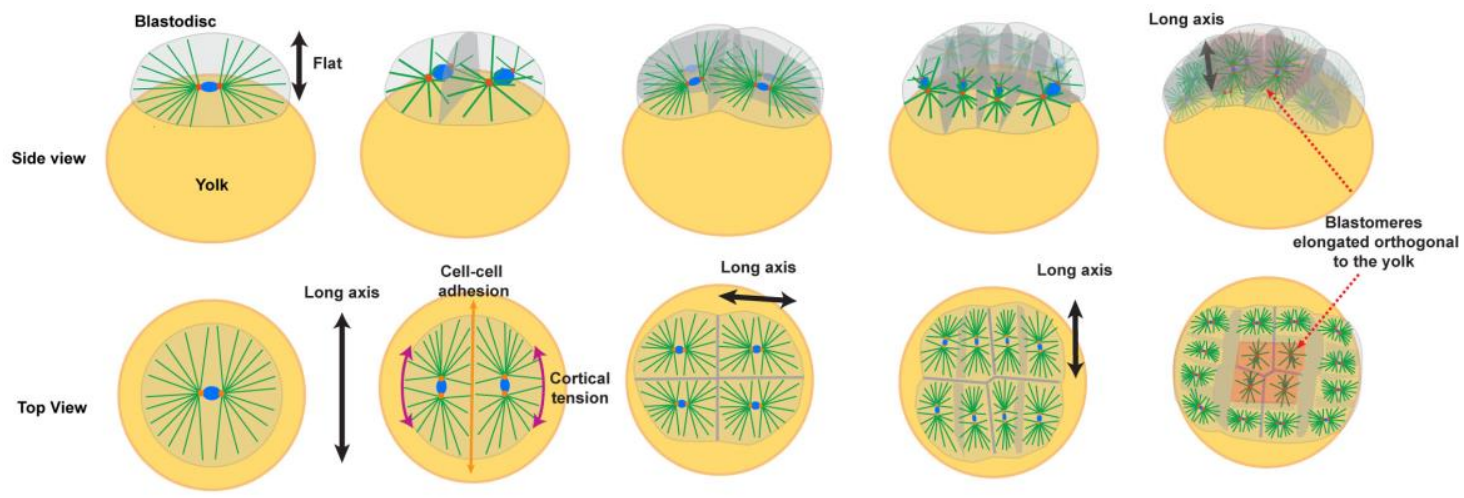

$\mathbf{F}$

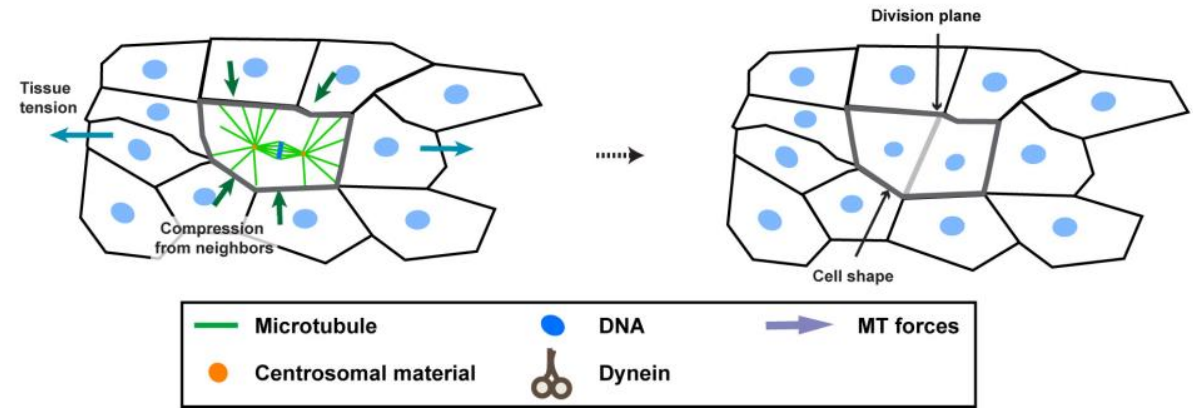


Fig. 3: Cell-shape sensing for division positioning. A) Astral Microtubule (MT) exert length-dependent forces to center nuclei and centrosomes. Length-dependency may arise from MT polymerizing and buckling or from pulling in the cytoplasm that is mediated by dyneincargo motion. B) Aster growth and length-dependent forces provide dynamic shape-sensing abilities to MT asters and result in aster-centering paths that depend on cell shape.

Reproduced with modifications from (Tanimoto et al., 2016). C) The cellular response to the application of external forces with magnetic tweezers on centered asters suggests that asters behave as elastic springs to maintain the spindle in the cell center. Red curve: Upon force application, the distance to the center, $d(t)$ increases and reaches a plateau value $d_{\max }$, at which aster forces balance the magnetic external force. When the force is released $d(t)$ relaxes exponentially to 0 . Blue curve: By varying the externally applied forces, and measuring the resultant value of $d_{\max }$, the stiffness of the centering spring can be computed. D) When an egg is shaped into a rectangular microwell, the torques and forces generated through lengthdependent MT forces will align nuclei or spindles with the long axis of a cell. E) Cleavage patterns of zebrafish embryos exemplify the iterative influence of cell shape on division orientation and vice versa. F) (Left) Cells in tissues may be exposed to external mechanical forces, such as tissue tension or compression from neighboring cells, which may influence cell shape and consequent spindle orientation with respect to external forces. (Rigth) As a consequence cells will divide with respect to those mechanical forces; which could in turn relax tissue stress, or influence the topology of cell-cell contacts. 